\title{
Rapid prototyping methods for the manufacture of fuel cells
}

\author{
Piotr Dudek1,a , Andrzej Raźniak² and Bartłomiej Lis ${ }^{2}$ \\ ${ }^{1}$ AGH University of Science and Technology, Faculty of Mechanical Engineering and Robotics, Al. A. Mickiewicza 30, 30-059 Kraków, \\ Poland \\ ${ }^{2}$ AGH University of Science and Technology, Faculty of Energy and Fuels, Al. A. Mickiewicza 30, 30-059 Kraków, Poland
}

\begin{abstract}
The term rapid prototyping (RP) is widely used to describe technologies which create physical prototypes directly from digital data. The first methods for rapid prototyping became available in the late 1980s and were used to produce models and prototype parts. Three-dimensional (3D) rapid prototyping methods are widely employed in various areas of manufacturing, research, and education. Typical applications include engineering, architecture, and medicine. The present level of technical and commercial demand requires the development of faster and cheaper methodologies for the design and execution of structures. Various techniques based on CAD platforms and rapid prototyping are available. Parts can be produced in a variety of materials: polymers, metal, paper, ceramics, and composites.

The RP method also enables the production of prototypes useful for analysing the characteristics of a complex system (e.g. interference between dynamic parts, geometric evaluation, quality, and reliability). RP technologies can produce very complex parts which are impossible or difficult to produce by traditional methods.

This paper presents typical applications of rapid prototyping technology for the manufacture of mechanical parts for fuel cells, such as housing parts or bipolar plates which supply reactants (hydrogen to anodes and oxygen to cathodes), conduct electrons from one cell to the next, remove waste heat from cells, and provide mechanical support for cells in a stack. Conventional graphite or composite materials can be replaced by lighter metallic materials characterised by vastly superior manufacturability and cost effectiveness, greater mechanical strength, increased durability and resistance to shock and vibration, and zero permeability.

The potential for the application of this method for the manufacture of metallic bipolar plates (BPP) for use in proton exchange membrane fuel cells (PEMFCs) is presented and discussed. Special attention is paid to the fabrication of light elements for the construction of PEMFC stacks designed for mobile applications such as aviation technology and unmanned aerial vehicles (UAVs).
\end{abstract}

\section{Introduction}

A traditional generator converts fuel to electricity by using machinery to convert thermal energy from combustion of the fuel into first mechanical and then electrical energy. A fuel cell, on the other hand, is a clean, quiet electrochemical device that converts hydrogen into electricity with no emissions (so-called 'zero emissions'). Today, while fuel cell technology is still considered pre-commercial, it offers significant economic and environmental benefits and is already being introduced in a number of applications. One of the critical steps in the commercialisation of fuel cells will be reducing the cost of the technology [1-3].

Rapid prototyping (RP) technologies can automatically manufacture near-net-shape parts with complicated geometry from computer-aided design (CAD) data. The 3D part is built up by means of powder consolidation, solidification of fluids, or gluing sheets in layers (the 'additive' or 'generative' process). For this reason, these techniques are often referred to as solid freeform fabrication or layered manufacturing $[4,5]$.

\footnotetext{
${ }^{\mathrm{a}}$ Corresponding author: pdudek@agh.edu.pl
}

For instance, in contrast to traditional 'subtractive' processes (e.g., drilling, milling, grinding), 'additive' RP methods enable the fabrication of products with complex geometries such as undercuts, internal cavities, or overhangs, as well as the generation of parts with oriented internal pore structures which cannot be manufactured using other approaches.

Due to this technology, it is possible to significantly shorten fabrication times and costs. Technologies such as selective laser sintering (SLS), direct metal laser sintering (DMLS), stereolithography (SLA), or fused deposition modelling (FDM) can be used for the fabrication of fuel cell prototypes, particularly for planar configuration.

It must be stated that RP technologies will never completely replace other manufacturing techniques, especially in large production batches, in which mass production is more economical. For short production runs, however, the cost of using RP is much lower, since no tooling is required. This technology is also ideal 
for the production of custom parts to meet the exact specifications of the user [6].

Rapid prototyping is beginning to change the way companies design and build products. The introduction of non-polymeric materials such as ceramics and composites enable RP users to produce functional, strong, and durable parts. The use of ceramics, metals, and composite materials greatly expands the range of products that can be made by means of rapid manufacturing .

Fuel cells are devices known for the most efficient electrochemical conversion of fuel into electrical energy. There are several papers showing the potential for using RP methods to fabricate elements for fuel cell with complicated shapes or housing characterised by reduced weight and improved heat dissipation designed for mobile applications such as aviation technology and unmanned aerial vehicles (UAVs) [1, 7-11].

\section{Definition and classification of rapid manufacturing}

All RP techniques employ the same basic process, as shown in Figure 1:

- Creating a design model and converting the CAD model to an STL format;

- Slicing the STL file into thin cross-sectional layers;

- Construction of a model, layer upon layer;

- Cleaning and finishing the model.

\section{Creating a CAD model and converting it to STL}

The first step is to create the object to be built using CAD software. This process is identical for all RP construction techniques. $\mathrm{CAD} / \mathrm{STL}$ parts can also be prepared using reverse engineering techniques using $3 \mathrm{D}$ scanners or $\mathrm{CT} / \mathrm{MRI}$ machines and appropriate software. It must be mentioned that following conversion to STL (stereolithography) format, some objects lose a certain degree of precision. STL is the standard file type used by most additive manufacturing systems. An STL file is a triangular representation of three-dimensional surface geometry. The surface is tessellated or broken down logically into a series of small facets (triangles). Each triangle is described by three points representing the vertices (corners) of the triangle and a direction perpendicular to it. These data are used by a slicing algorithm to determine the cross sections of the 3D shape to be built by the rapid prototyping machine.

\section{Slicing the STL file into thin cross-sectional layers}

In this step, a slicing program processes the STL file in order to create slices of the part to be printed. Several programs are available, most of which are incorporated into printer control software, enabling the user to adjust the size, location and orientation of the model. The orientation is important for several reasons. First, the properties of prototypes vary with the direction of the coordinate. For example, prototypes are usually less accurate and weaker in the $z$ direction (vertical) than in the $x-y$ (horizontal) plane. Furthermore, the orientation of the part partially determines the period of time required to build the model. Placing the shorter dimension in the $z$ direction reduces the number of layers needed, thereby reducing construction time. The program can also generate an auxiliary structure to support the model during the building process, which is necessary for some technologies.

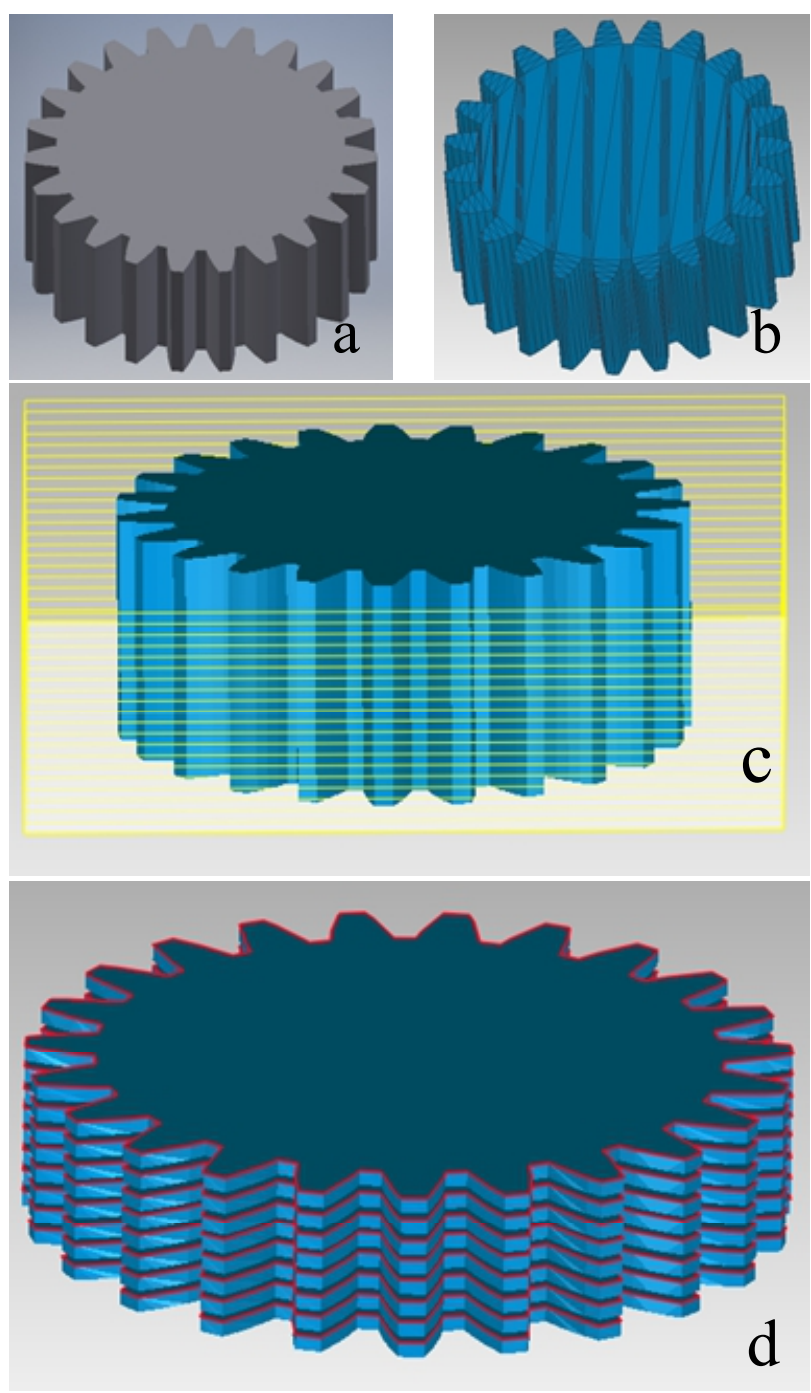

Figure 1. Schema of RP methods ( $a$ - a CAD model, $b$ - the model is converted to STL triangles, $\mathrm{c}-$ the model is sliced, $\mathrm{d}-$ the model is built layer by layer).

\section{Building the layered model}

The next step is the physical construction of the part. Using one of several techniques (described in the next section), an RP machine builds one layer at a time from a polymer, paper, metal, plastic powder, or other starting material. Most machines are fairly autonomous, requiring little human intervention.

\section{Cleaning and finishing the model}

The final step is post-processing work, which involves removal the prototype from the machine, separation of some supports, cleaning and removal of unused powder, and additional hardening of the final parts. Some technologies require special procedures for the proper manufacture of a part. For example, photosensitive materials need to be cured completely before use, while other technologies require cleaning and surface treatments such as sanding. 
There are various ways to classify rapid prototyping technologies; one is based on the form of the starting material (Figure 2). In this chapter, some of the most important technologies are presented.

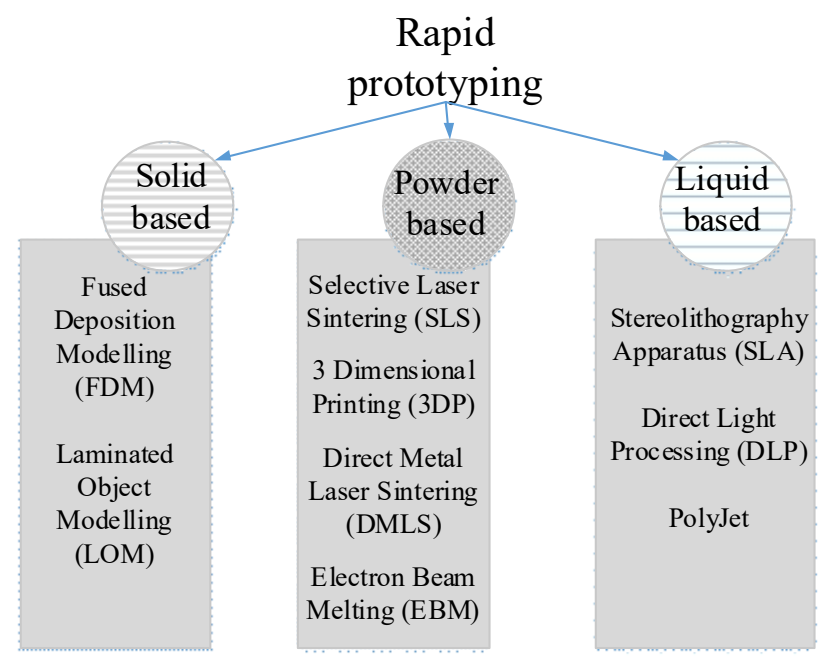

Figure. 2. Schema of RP methods.

\subsection{Stereolithography}

This process, patented as a means of rapid prototyping in 1986 by Charles Hull, co-founder of 3D Systems, Inc., initiated the rapid prototyping revolution. In this process, a uniquely designed $3 \mathrm{D}$ printing machine called a stereolithograph apparatus (SLA) converts liquid plastic into solid objects.

SLAs have four main parts: a tank filled with liquid photopolymer, a perforated platform that is lowered into the tank, an ultraviolet laser, and a computer controlling the platform and the laser.

As shown in Figure 3, the model is built on a platform located under the surface in a liquid photopolymer. A low-power laser traces the first layer, solidifying the first cross section of the model.

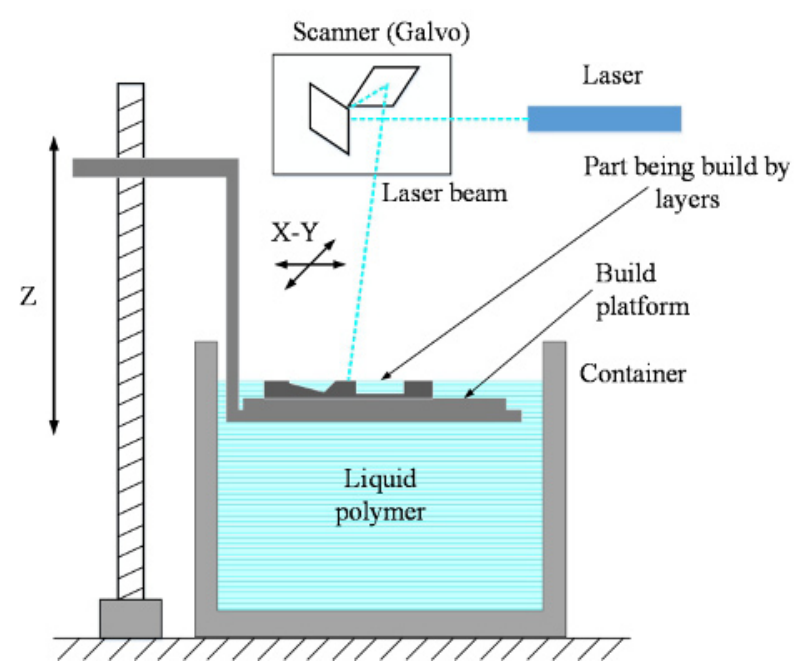

Figure 3. The stereolithography (SLA) process.

Once the initial layer has hardened, the platform is lowered, exposing a new surface layer of liquid polymer. The laser traces the next cross section of the object being printed, which instantly bonds to the hardened section beneath it. This process is repeated again and again until the entire object has been formed and is fully submerged in the tank. The platform is then raised to expose a three-dimensional object. After being rinsed with a liquid solvent to free it of excess resin, the object is baked in an ultraviolet oven to further cure the plastic; subsequently, any supports are broken away.

Currently available photocurable materials include acrylic and epoxy resins . Researchers are investigating the possibility of manufacturing parts reinforced with carbon fibres. Additionally, ceramic powders can be added to UV curable resins so as to form a so-called 'green' part, and then thermally treated to eliminate the resin and sinter the part. Objects made using stereolithography are generally characterised by smooth surfaces, but the quality of the object depends on that of the SLA machine used to print it.

\subsection{Fused deposition modelling}

Fused deposition modelling (FDM) is one of the bestknown methods of rapid prototyping. A thermoplastic filament is unwound from a coil and material is supplied to an extrusion nozzle, assembled to a mechanical head which can be moved over a table according to the required slice geometry, which deposits a thin bead of extruded plastic to form each succeeding layer. The nozzle is heated to the melting point of the plastic and includes a mechanism which enables the flow of melted plastic to be stopped or continued. The result of lamination of the solidified material to the preceding layer is a plastic $3 \mathrm{D}$ model built up one layer at a time (Figure 4).

FDM printers use two kinds of materials: modelling material which constitutes the finished object, and support material which acts as a scaffolding to support the object as it is being printed. Once the part is completed, the support columns are removed and the surface is finished.

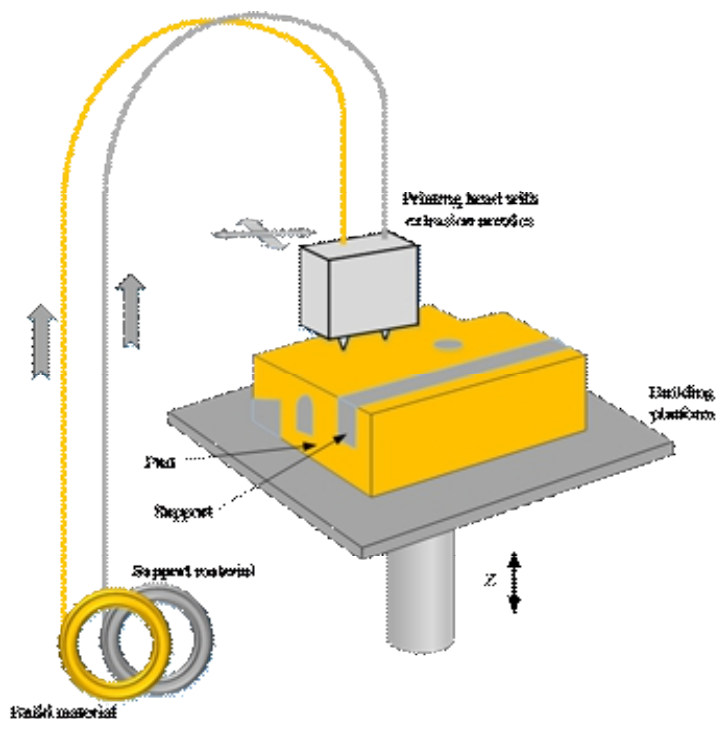

Figure 4. The FDM process.

\subsection{Selective laser sintering}

The SLS process was developed and patented in the 1980s by Carl Deckard, then an undergraduate 
student at the University of Texas, and his mechanical engineering professor, Joe Beaman. Objects printed using SLS are made with powder materials, most commonly plastics such as nylon, which are dispersed in a thin layer on top of the building platform inside an SLS machine. A computer-controlled laser sends pulses down to the platform, tracing a cross section of the object onto the powder (Figure 5).

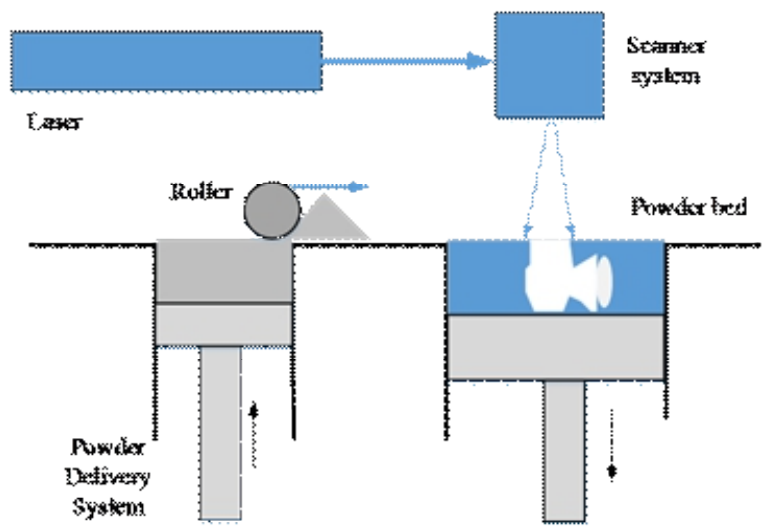

Figure 5. The selective laser sintering process.

The platform is lowered to the height of the next layer and powder is reapplied. The process continues until the part is complete. Excess powder in each layer helps support the part during construction. The process chamber is heated to prevent part warping and filled with nitrogen to reduce powder aging. When the object is fully formed, it is left to cool in the machine before being removed.

The materials available for SLS technology include polyamide, polyethylene, elastomers, ceramics, and metals, using polymers as additives. This technique is still in development for ceramic materials, due to difficulties arising from sintering; however, structures can be fully densified via sintering in the liquid phase.

\subsection{Direct metal laser sintering/selective laser melting}

Like SLS, this technology employs a laser as a way to consolidate powder, but uses metal powder as source material.

Preparation for a build requires support, even though parts are sintered in a bed of powder (as in SLS). The rapid melting of each metal layer can create residual stresses in the part during DMLS. Supports are required to hold parts in place in order to prevent any warping that might occur from these stresses as each layer cools.

DMLS uses a laser system that draws onto a surface of atomised metal powder. Using a focussed laser beam, this technology melts metal powder locally to fuse it into solid parts. Upon the completion of each layer, a blade adds a fresh layer of powder and repeats the process until the final metal part is formed (Figure 6).

Once the build is finished, it is necessary to remove the support structures and carry out any other postprocessing work required; for example, surfaces which were in contact with support structures must be cleaned, and any bead blasting and deburring needed to finish the parts must be carried out.
In SLM, the material is fully melted, whereas in DMLS, the material is heated just enough for the molecules to fuse. Both technologies are capable of producing parts of over $98 \%$ density. Leftover powder can be reused repeatedly, so waste levels are low.

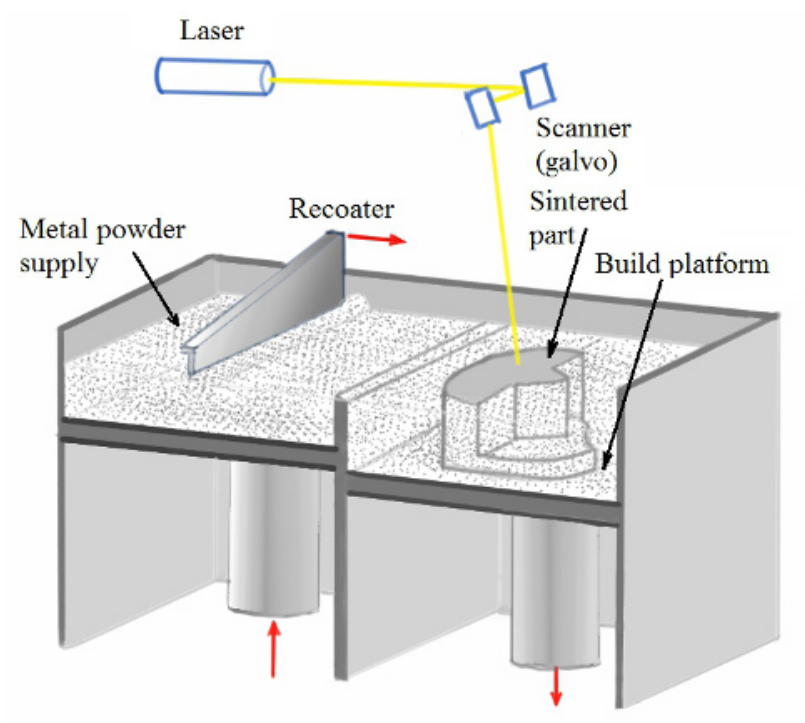

Figure 6. The DMLS process.

Compared to SLS, this technology has different requirements for the design of parts, and also special requirements for positioning parts on the platform.

\section{Application of 3D printing for manufacturing some components of PEMFC fuel cells}

At present, proton exchange membrane fuel cell (PEMFC) technology shows a relatively high level of development. Various energy power systems based on PEMFC technology are already available in the marketplace. They can be used as power generators with a wide range of electric power from 5 We to 200 $\mathrm{kWe}$, from stationary and military to automotive and aviation applications, for example: (i) the small (5 We, cooled by heat spreaders) and portable Minipak mini charger from the Horizon company, for charging USBcompatible portable devices [12]; (ii) Panasonic 750-We domestic CHP plants with PEMFCs (hydrogen from reformed gas) supplying electricity and hot water [13]; (iii) Toyota Mirai, the first mass-produced car to run on hydrogen, using a TFCS (Toyota Fuel Cell System) with a capacity of $114 \mathrm{~kW}$, powered by hydrogen derived from composite hydrogen tanks at a pressure of 700 bar [14].

A proton exchange membrane fuel cell (PEMFC) is an electrochemical device in which hydrogen and oxygen react electrochemically, resulting in the generation of electricity (up to $55 \%$ based on the high heating value of hydrogen), heat, and water [3].

The membrane, which acts as the electrolyte, is compressed between two porous, electrically conducting electrodes, typically made out of carbon cloth or carbon fibre paper. At the interphase between the porous electrode and the polymer membrane is a layer 
with catalyst particles, typically carbon-supported platinum $[2,3]$.

One of the challenges involved in commercialisation is finding a new method of fabrication which would enable the production of reproducible components at reduced costs [2].

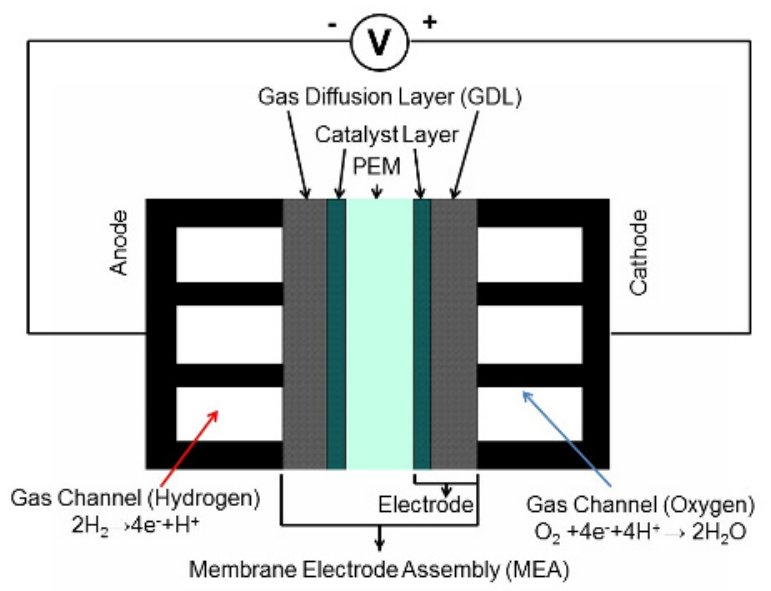

Figure. 7. The idea behind the construction of a single PEMFC fuel cell.

Some examples of the application of injection printing technology to the fabrication of Nafion polymer electrolytes exist. This technique can substitute for commonly-used membrane foil. A Nafion ${ }^{\circledR}$ dispersion is deposited directly onto the catalyst layers of anode and cathode gas diffusion electrodes. The two electrodes are then pressed together with their membrane layers facing each other. For the manufacture of PEMFC components it is necessary to precisely deposit a very thin layer of platinum, needed for the oxidation and reduction reactions, with a high level of utilisation efficiency. This can be critical in terms of taking this technology to the masses. Another process is screen printing, but this is done by hand and necessitates compromises in terms of uniformity and time efficiency. The process is greatly improved by using the $3 \mathrm{D}$ printing process to deposit the layer of platinum. The inkjet printing method is 4 to 5 times faster than screen printing $[8,11]$.

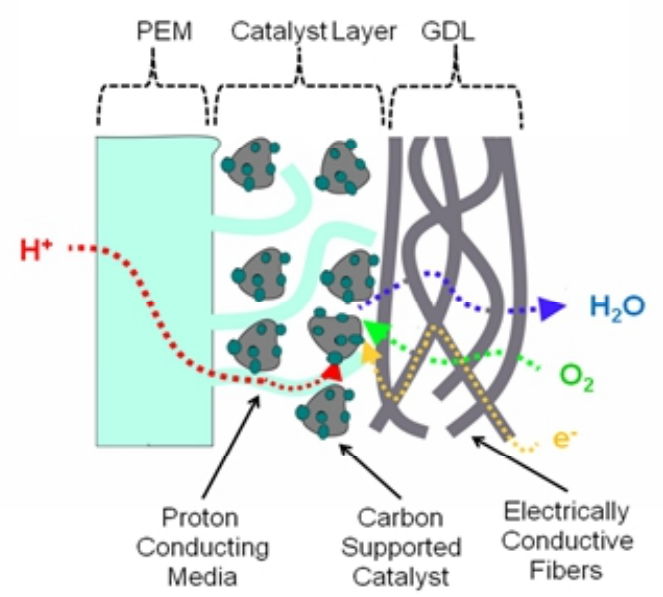

Figure 8. The idea behind MEA construction and operation.
The main parameters that determine the application of a single membrane in a PEMFC designed stack are power output, stability of electrical parameters under load, and water management. Water is necessary in order to hydrate the membrane, increasing its electrical conductivity. However, in excess, water can flood the pores of the GDL (gas diffusion layer) and hinder the reactants from reaching the active centres of the catalyst layer. Thus, an efficient balance between generated and removed water is necessary to obtain good performance from a PEMFC. Adequate hydration in the PEM and GDL enables the attainment of higher current values, increasing the power provided by the PEMFC [9].

The distribution of gaseous reagents is also a crucial parameter determining the power output of a singlemembrane PEMFC stack. Special architecture of the gas diffusion layer can lead to enhanced performance by a PEMFC. It was found that the performance of a single MEA was enhanced by the utilisation of microchannels $(0.05-1 \mathrm{~mm})$ to improve gas routing. Tests performed on distributed water in actual fuel cell microchannels microfabricated using the stereolithography technique seem to confirm these statements. Side channels or slots perpendicular to the main microchannel are used in the microfabricated samples, whereas the stereolithography samples incorporate a sandwiched GDL for distributed water injection into the main channel $[15,16]$

A PEMFC stack consists of a number of single MEA cells stacked so that the cathode of one cell is electrically connected to the anode of the adjacent cell. The key aspects of fuel cell stack design are: uniform distribution of reactants to cells and within each cell; maintenance of required temperature in each cell; a minimum of resistive losses (choice of materials, configuration, uniform contact pressure). A special role is played by bipolar plates, which are the backbone of a PEMFC stack. They act as current collectors in the fuel cell environment and provide conduits for the reactant gases. Bipolar plates are usually fabricated from graphite and metal. In special application in unmanaged aerial vehicles (UAV) technology, the mass of the entire system is very important. The capacity for hydrogen storage is also critical for UAV application, as flight duration depends on it. Graphite bipolar plates are typically used in PEMFC stacks designed for stationary applications, whereas in the case of PEMFC applications in UAVs and transports, mass seems to be one of the crucial parameters. Some data in the literature indicate that the application of metallic bipolar plates enables not only a reduction in mass but also relative savings in hydrogen consumption. Comparative results using bipolar plates made from graphite and metal indicated that hydrogen consumption decreased by at least of $18 \%$ due to higher electrical and thermal conductivity $[10,17]$.

\section{Application of 3D printing for manufacturing air inlet channel}

The design and execution of a PEMFC stack as a basic unit to power an electric motor in an unmanned aerial vehicle requires knowledge of certain electrical 
characteristics: the voltage (U)-current (I), and power (P)-current(I) curves produced by the DC power sources. Another requirement is adjustment of the operating parameters of the PEMFC stack to the scope of permissible values of current and voltage consumed by the electric motor. In the case of aeronautical applications, it is also crucial not to exceed the permissible mass of a PEMFC stack with a hydrogen fuel tank.

Even though products dedicated to unmanned aerial vehicle applications are already available on the fuel cell market, it is not always possible to use a commercial product due to the specific design solutions needed for UAV construction. At AGH University of Science and Technology, Faculty of Fuels and Energy, research was undertaken to adapt a commercial 300-W PEMFC stack (Horizon Singapore) to an electrical engine in order to supply a UAV. It should be emphasised that this route has also been taken by most of the leading R\&D centres dealing with applications of fuel cell technology in aerial vehicles $[18,19]$. Our team planned each step of design changes aimed at reducing the weight of the generator by: i) removing the metal casing and replacing it with a structure made of 3D-printed plastics; ii) removing an electronic circuit used to power fans and to humidify and purify the PEMFC stack; iii) eliminating fans and replacing them with air jets to cool the stack. Figure 9 shows examples of 3D-printed models of supply channels for the air jets aimed at reducing the number of fans, which not only increase the weight of the system but also draw power from the fuel cell stack.

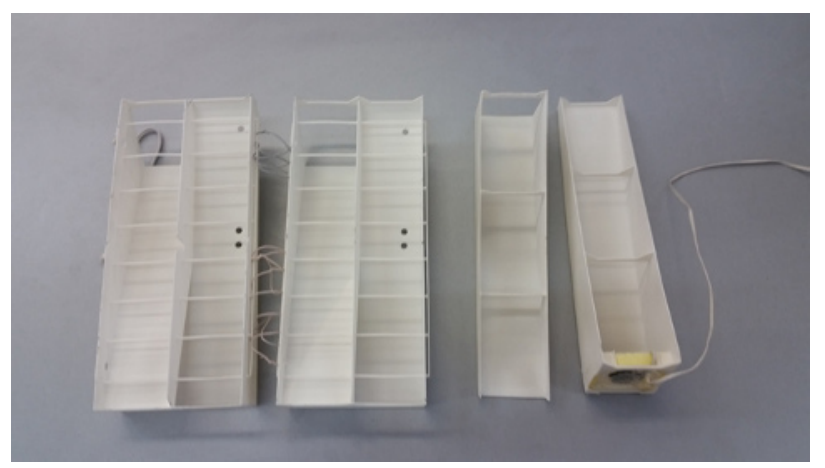

Figure 9. The examples of 3D printed models.

Figure 10 presents a photo of an experimental setup designed for investigations of a 300-W PEMFC stack, including an air jack fabricated by means of FDM technology. The main parts of the experimental setup comprised an electrical engine with a propeller, an electronic load, and a model of the fuselage manufactured from styrofoam.

The main function of this model was to reflect the actual fuselage constructed by the UAV producer. In the course of the experimental investigations, aimed at determining the suitability of the 3D-printed air jack, the electric motor was supplied from an external DC source. The air flow, driven directly into the tight channel, passed through the PEMFC fuel cell stack and exited the fuselage. The speed of the air flow directed to the inlet of the fuselage was $24 \mathrm{~km} / \mathrm{h}$. Under these conditions, the characteristics of the voltage (U)-current (I) and power (P)-current (I) curves were determined.

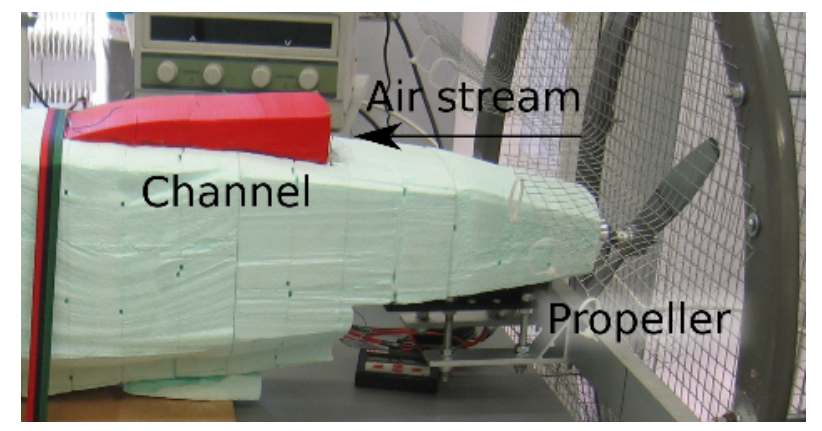

Figure 10. Photo of an experimental setup designed for investigations of a 300-W PEMFC stack with air inlet channel.

Concurrent temperature measurements were taken at points $\mathrm{T}_{1}$ and $\mathrm{T}_{2}$ using thermocouples placed at different locations in the fuselage. Thermocouple 1 $\left(\mathrm{T}_{1}\right)$ was placed inside the front part of the stack; the second thermocouple $\left(\mathrm{T}_{2}\right)$ was placed in the middle of the PEMFC stack, which was, in turn, placed in the fuselage. To avoid any unexpected temperature increases inside the PEMFC stack during operation, the fuel cell was gradually loaded (first part of Fig. 11). It was established that the maximum temperature of the stack would not be allowed to rise above $55^{\circ} \mathrm{C}$. In Figure 11 , variations in power $(\mathrm{P})$ and temperature $\mathrm{T} 1, \mathrm{~T} 2$ of the PEMFC stack vs time was analysed. As can be seen, the PEMFC stack attained about $200 \mathrm{~W}$ of electrical power. No significant difference was observed between $\mathrm{T} 1$ and $\mathrm{T} 2$. At $2300 \mathrm{~s}$, the electronic load was switched off. In the second part, the PEMFC stack was fast-loaded to reach $200 \mathrm{~W}$. In this part of the experiment as well, the temperature inside the PEMFC reached values no higher than $55^{\circ} \mathrm{C}$. Based on these preliminary results, it seems important to carry out further research on the analysis of the parameters of air flow and the construction of the channel.
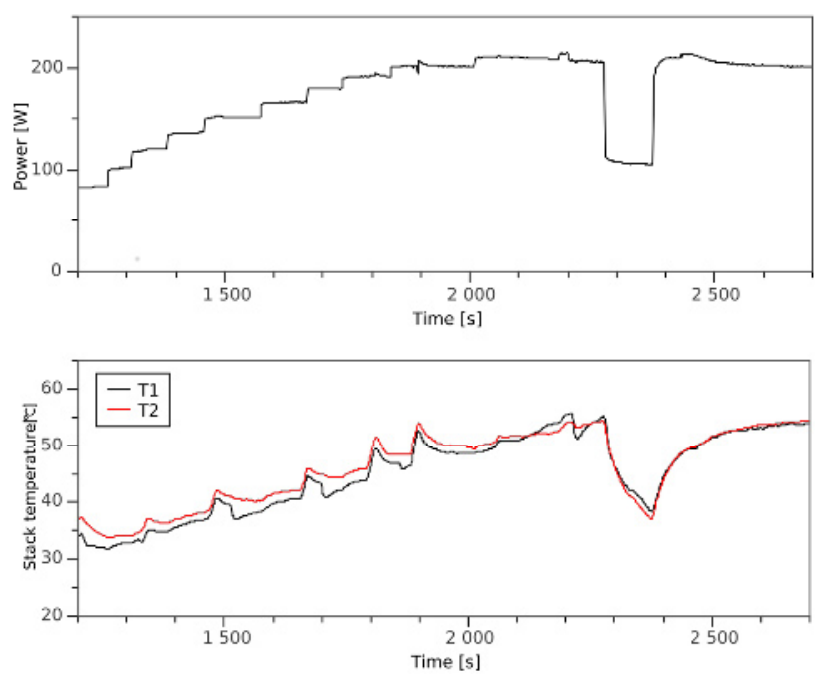

Figure 11. Performance of a PEMFC stack with a tested 3Dprinted channel. 
The concept of reverse engineering is defined as follows by Bradley and Currie [4]: 'the process of duplicating an existing part, subassembly, or product without drawings, documentation, or a computer model is known as reverse engineering. Reverse engineering is also defined as the process of obtaining a geometric CAD model from 3D points acquired by scanning/digitising existing parts/products'.

Sometimes it is necessary for some reason to change a commercial product to meet the requirements of specific solutions. In this case it was impossible to order parts or a solution that fit our requirements; therefore it was necessary to modify the product.

Due to these requirements, research was performed at the Department of Manufacturing Systems at AGH to develop lightweight housing elements for PEMFCs. These elements were required to be mechanically strong, as rigid as the original, and characterised by good thermal conductivity for appropriate heat dissipation.

In order to develop these elements, a measurement arm with an integrated scanning head was used. A scanned 3D housing element was processed using reverse engineering programs to create $\mathrm{CAD}$ elements, which were then subjected to additional processes in order to reduce their weight (Figure 12). Weight reduction in housing parts without a commensurate reduction in strength is very important for the use of fuel cells in aerospace applications. Additive Manufacturing (AM) affords the flexibility to create complex part geometries that are difficult to build using traditional manufacturing. It can build parts that incorporate design features such as internal cavities, pipes, and lattice structures that help to reduce the weight of parts without compromising their mechanical performance. Furthermore, AM machines produce less scrap than traditional machines, a critical attribute when using expensive aerospace materials; thus this process enables the use of materials such as titanium at an acceptable cost.

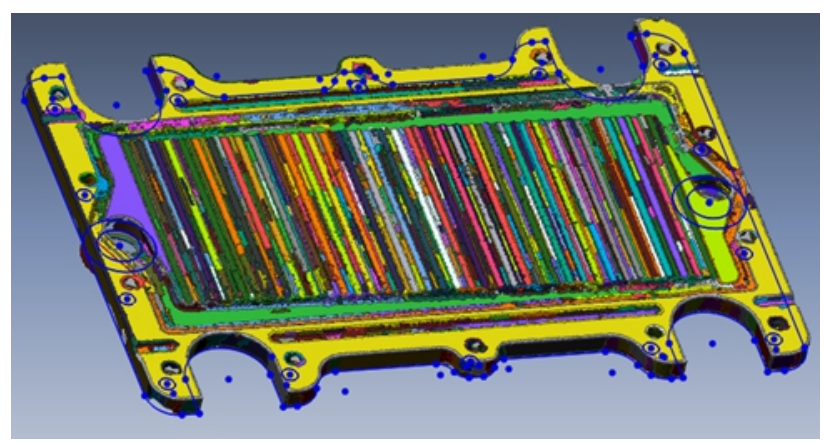

Figure 12. Scanned housing element of a PEMFC stack in a reverse engineering application.

After the necessary research, new PEMFC housing elements were created. In the next stage, CAD parts were converted to STL format and printed on an EOS Formiga P 100 SLS (stereolithography) machine using the polyamide powder PA2200 (also known as PA12). Printed parts (original project based on scanned elements and developed new) were compared in order to find differences in stress and strength between the old and new elements. Cooling channels were integrated into the housing, reducing the risk of damage to the PEMFC due to a coolant leak.

\section{Conclusions}

Rapid prototyping is characterized as a low-cost manufacturing process with high flexibility for the production of functional prototypes and customized products. Its working principle is based on the imposition of layers forming a three-dimensional design of varying complexity, using various techniques. There is an increasing interest in the polymer fuel cell technology for constructing new reliable power sources for stationary, transport, avionic, and military applications. One of main goals of the PEMFC technology is lowering manufacturing costs for continuous production. The conventional fuel cell manufacturing processes requires long time and high costs, especially in the stages of product development and optimization. The techniques of Selective Laser Sintering (SLS), Laminated Object Manufacturing (LOM), and Three Dimensional Printing (3DP) may be considered as promising alternatives for the production of fuel cell prototypes. SLS, despite the high initial cost of the equipment, can conform and sinter the components simultaneously. LOM uses tapes, produced by tape casting, to obtain laminates. 3DP already has the lowest cost of acquisition of equipment and manufacturing of the prototype. Compared to other methods, RP can produce very complex parts which are impossible or difficult to manufacture by traditional methods. PEMFC tackles niche applications in military and aviation, which require the special construction of PEMFC stacks as they need to fit to the entire electrical system. In case of UAVs, the weight of the power source is also a critical parameter. The replacement of fans with a 3D-printed channel allows for mass reduction, and limits electric power consumption for PEMFC stack cooling. Cooling possibilities of air taken from stream located behind the propeller are proportional to fuel cell load by the main electric motor. Further studies will concentrate on the design of the channel shape and structure, which will ensure the effective cooling the elements in the PEMFC stack.

\section{Acknowledgements}

The work was carried out under the project no. PBS3/A6/24/2015 The National Centre for Research and Development (2015-2018)

\section{References}

1. T.H. Bradley, B.A. Moffitt, D. Mavris, D.E. Parekh, Applications - Transportation Aviation: fuel cells in: Reference Module In Chemistry, Molecular Sciences And Chemical Engineering Encyclopedia Of Electrochemical Power Sources, (Elsevier 2009)

2. Y. Wang, K.S. Chen, J. Mishler, S.C. Cho, X.C. Adroher, Applied Energy, 88 (2011)

3. D. Stolten, Hydrogen and Fuel Cells: Fundamentals, Technologies and Applications (John Wiley \& Sons, 2010) 
4. C. Bradley, B. Currie, Computer-Aided Design \& Applications 2, 5 (2005)

5. J. P. Kruth, CIRP Annals-Manufacturing Technology 40, 2 (1991)

6. I. Gibson, D.W. Rosen, B. Stucker, Additive manufacturing technologies: rapid prototyping to direct digital manufacturing, (New York, 2010)

7. O. Z. Sharaf, M. F. Orhan, Renewable and Sustainable Energy Reviews 32 (2014)

8. M. Klingele, M. Breitwieser, R. Zengerleab, S.Thieleac, J. Mater. Chem. A 3 (2015)

9. J.A. Salva, A. Iranzoa, F. Rosab, E. Tapia Energy 101 (2016)

10. B.D. Gould, J.A. Rodgers, M. Schuette, K. Bethune, S. Louis, R. Rocheleau, K. Swider-Lyons, J. Solid State Sci. Technol. 4 (2015)

11. Chen-Yu Chen, Yun-Che Wen, Wei-Hsiang Lai, Ming-Chang Chou, Biing-Jyh Weng, Ching-Yuan Hsieh and Chien-Chih Kung, J. Fuel Cell Sci. Technol. 8 (2010) 12. http://www.horizonfuelcell.com/\#!minipak/c156u (2016-05-07)

13. http://www.hvacr.pl/panasonic-opracowal-domoweogniwo-paliwowe-ene-farm-209 (2016-05-07)

14. https://www.toyota.pl/new-cars/mirai (2016-05-06)

15. https://gcep.stanford.edu/pdfs/SI3U6jOMPAIgwkai BD_77Q/goodson_ers06.pdf (12.05.2016)

16. C. H. Hidrovo, T.A. Kramer, E.N. Wang, S. Vigneron, J.E. Steinbrenner, Jae-Mo Koo, Fu-Min Wang, D.W. Fogg, R.D. Flynn, Eon Soo Lee, C.H. Cheng, T.W. Kenny, J.K. Eaton, K.E. Goodson, Heat Transfer Engineering, 27 (2006)

17. B. Lyons, M. Batalov, P. Mohanty, S. Das, Rapid Prototyping of PEM Fuel Cell Bi-polar Plates using 3D Printing and Thermal Spray Deposition, Proc. SFF Symposium, Austin, TX (2005)

18. J.M. Krawczyk, A.M. Mazur, T. Sasin, A.W. Stokłosa, Transactions of the Institute of Aviation 4 (2014)

19. O. Gonzalez-Espasandin, T.J. Leo, E. NavarroArevalo, The Scientific World Journal 2014, 497642 (2014) 